\title{
Méthodes de lutte contre les Loranthaceae
}

\section{Joseph I. Boussim et Nayéré Médah}

Résumé: Les Loranthaceae posent un problème majeur à l'amélioration et à la pérennisation de la production fruitière du karité (Vitellaria paradoxa C. F. Gaertn) en Afrique de l'Ouest. Cet'article présente les resultats des investigations faites en vue d'identifier des méthodes de contrôle des parasites. Deux types d'essais "au champ" ont été effectués et ont donné des résultats prometteurs: la destruction mécanique et les traitements par herbicides. Additionellement ont été identifiées des variétés résistantes et quelques possibilités de la lutte biologique.

Mots clés: Agelanthus, herbicides, lutte biologique, Tapinanthus, Vitellaria paradoxa

\section{Methodes to Fight against the Loranthaceae}

Summary: The Loranthaceae pose a major problem to the improvement and to the perpetuation of the fruit production of the shea nut (Vitellaria paradoxa C. F. Gaertn) in West Africa. In this article, the results of some investigations made in order to identify methods of control of the parasites are presented. Two methodes were tested in the field and gave promising results: mechanical destruction, and application of herbicides. Additionally we looked for resistent varieties, and for possibilities of biological pest control.

key words: Agelanthus, biological pest control, herbicides, Tapinanthus, Vitellaria paradoxa

\section{Methoden zUR BeKÄMPfung deR LoRANTHACEAE}

Zusammenfassung: Die Loranthaceae stellen ein großes Problem bezüglich der Aufrechterhaltung und Verbesserung der Fruchtproduktion des Schibutterbaunmes (Vitellaria paradoxa C. F. Gaertn) dar. Dieser Arikel präsentiert die Ergebnisse von Untersuchungen zur Bekämpfung dieser Parasiten. Zwei Methoden wurden erfolgversprechend im Feld getestet: mechanische Bekämpfung und Einsatz von Herbiziden. Zusätzlich suchten wir nach resistenten Varietäten und nach Möglichkeiten der biologischen Schädlingsbekämpfung.

Schlagworte: Agelanthus, biologische Schädlingsbekämpfung, herbicides, Tapinanthus, Vitellaria paradoxa

\section{INTRODUCTION}

Les Loranthaceae causent d'importants dégâts dans les formations naturelles et les plantations de nombreux pays africains : Burkina Faso, Cameroun, Côte d'Ivoire, Gabon, Ghana, Mali, Nigeria, Ouganda (RoOM 1970, Clerk 1978, SAllé et al. 1990, Boussim 1991, Boussim et al. 1993a et 1993b, Boussim 2002, Traoré 1994, Traoré \& DA 1995, 1996 et 1997, Guyot \& NTAWANGA OMANDA 1998) et du monde. Les dégâts, difficiles à évaluer, posent parfois un problème majeur dans la sylviculture. En Afrique de l'Ouest, le karité est l'une des essences naturelles les plus gravement atteintes. Les travaux de MAïGA (1988 et 1989) et de Boussim (1991) ont révélé qu'environ 95\% des karités du Mali et du Burkina Faso sont parasités par cinq espèces de Loranthaceae (Agelanthus dodoneifolius (DC.) Polh. et Wiens, Tapinanthus bangwensis (Engl. et K. Krause) Danser, T. globiferus (A. Rich.) Tieghem, T. ophiodes (Sprague) Danser et T. pentagonia (DC.) Tieghem). Bien avant ces résultats, RUYSSEN (1957) faisait remarquer que le contrôle des Loranthaceae est un préalable à l'amélioration du karité. C'est dire, si besoin en était, que la nécessité de lutter contre le parasitisme des Loranthaceae en Afrique n'est pas une nouveauté. En 1991, nous terminions un mémoire sur le parasitisme des Tapinanthus sur le karité en souhaitant qu'une méthode de contrôle des Loranthaceae dont plusieurs espèces affectent gravement nos parcs agroforestiers et vergers, soit trouvée à court ou moyen terme.

Le présent travail teste des méthodes de lutte classiquement reservées aux parasites végétaux herbacés des racines tels que les Striga et les Orobanches (DJIGUEMDÉ 1985, MinINGOU 1985, COMPAORÉ 1986, OuÉDRAOGO 1995, YonLI 1999, TRAORÉ 1999), afin de proposer celles qui sont applicables aux Loranthaceae.

\section{MÉTHOde}

Dans le cadre du présent travail, nous avons testé trois techniques de lutte couramment utilisées contre les mauvaises herbes et les plantes parasites : la destruction mécanique, les traitements par herbicides, l'identification des variétes résistantes.

\subsection{Lutte mécanique}

La lutte mécanique consiste à détruire manuellement le parasite. Depuis que nous avons pris conscience du problème des Loranthaceae, et compte tenu de l'absence de données sur les méthodes de lutte dans le cas du couple karité/Loranthaceae, nous avons préconisé la destruction directe des touffes parasites. Dans le souci de maximiser les chances de réussite de la technique là où elle est applicable et éviter des repousses, nous avons entrepris des essais de coupe à l'échelle de l'arbre et portant sur les touffes parasites. Les essais sont réalisés dans le peuplement de karité de Toudoubwéogo, un des villages riverains de Ouagadougou. Dans un champ, un karité fortement infesté par Agelanthus dodoneifolius et Tapinanthus globiferus a été repéré en avril, période ou l'arbre a perdu toutes ses feuilles permettant de 
voir, même de loin, le feuillage sympervirent des parasites. Le paysan propriétaire du champ reconnaît que l'arbre ne produit plus beaucoup et pense que le parasitisme en est la cause. Aussi, lui avions-nous proposé de procéder à un ébranchage de l'arbre afin de le débarrasser des parasites et de tester les capacités de repousse du karité. L'arbre a été émondé en juillet (Fig. 1).

Deux karités parasités à la fois par A. dodoneifolius, T. globiferus et $T$. ophiodes sont retenus dans le même champ pour les essais de coupe des touffes parasites. 24 touffes (6 par parasite) ont été choisies en fonction de leur accessibilité. Les traitements sont appliqués en trois temps : 9 touffes sont coupées le 24 avril (reprise de la végétation), 9 autres le 15 juin (pleine période végétative) et 6 autres le 13 novembre (début de la période de ralentissement de l'activité végétative). Les tiges constituant les touffes sont comptées, puis coupées à la base (Fig. 2). Les relevés, qui consistent à compter les repousses, ont lieu toutes les 3 semaines.

Parallèlement à la coupe de la partie aérienne en épargnant le système endophytique du parasite, 6 rameaux de karité portant des touffes parasites sont coupés en amont du point de fixation du parasite.

\subsection{Lutte chimique}

A l'instar des parasites épirhizes, des essais de lutte chimique sur trois Loranthaceae sur karité ont été effectués dans la station de recherche de Saria, à Koudougou. Deux herbicides systémiques sont choisis pour les tests. Ce sont :

- Le calliherbe dont la substance active est l'acide 2,4-Dichlorophénoxyacétique ou 2,4-D.

- Le Kalach ou Roundup ayant pour substance active le glyphosate ou 2-N- phosphonométhylglycine.

Le choix de ces produits a été guidé par les résultats probants qu'ils ont permis d'obtenir dans les essais de lutte contre le gui des feuillus (Frochot \& Delabraze 1979, Frochot et al. 1983, BAillon et al. 1988, SAllé et al. 1993). Les doses de 15, 20, 25, 35 et $45 \mathrm{~g} / 1$ (T1, T2, T3, T4, T5) sont retenues et appliquées aux trois espèces parasites: A. dodoneifolius, T. globiferus, T. ophiodes. Les touffes de parasites sont regroupées en trois lots : Lot $1=$ témoin; Lot 2 = un seul traitement; Lot $3=$ deux traitements avec un intervalle de deux semaines.

Le traitement est appliqué par aspersion foliaire jusqu'à début de ruissellement. Les symptômes et la quantité de parasite détruite selon les doses pour chacun des deux herbicides sont notés hebdomadairement.

\subsection{Résistance variétale}

Dans le même ordre d'idée, l'identification des arbres résistants nous paraît une voie prospectable. Chez les céréales, l'existence de différences de sensibilité de plusieurs cultivars de sorgho au Striga hermonthica a été mise en évidence (Dogget 1952, 1965, Last 1960a in Olivier 1996). Chez le peuplier, HARIRI (1989) et HARIRI et al. (1990) rapportent l'existence de cultivars résistants au gui.

$\mathrm{Au}$ cours des sorties sur le terrain, nous avons observé des karités sans parasite cohabitant pourtant avec des arbres fortement infestés, ce qui présume une différence de sensibilité au parasite. Pour vérifier l'effectivité de la résistance, nous avons entrepris des essais d'infestation artificielle sur quelques-uns de ces arbres sains. Cinq pieds adultes de ces arbres non parasités ont été repérés dans le parc de Toudoubwéogo et infestés chacun en janvier 1999 par 60 baies d'A. dodoneifolius et en avril par 60 baies de T. globiferus. L'année suivante, les essais ont été repris sur les mêmes arbres, à la même période et avec les mêmes quantités de baies.

\subsection{Lutte biologique}

La bibliographie disponible ne donnant aucun renseignement sur les ennemis naturels des Loranthaceae, nous avons entrepris de rechercher, dans la perspective de lutte biologique, d'éventuels agents pathogènes, insectes et autres animaux, qui affecteraient les Loranthaceae en conditions naturelles.

\section{RÉSULTATS}

\subsection{Lutte mécanique}

L'ébranchage complet de l'arbre a eu pour effet l'élimination totale du parasite. Dès la troisième année, l'arbre a retrouvé un niveau satisfaisant de repousse et un début de floraison. La production fruitière a été nettement améliorée à partir de la cinquième année (Fig. 1).

La destruction partielle de la touffe parasite, en épargnant le système endophytique, est suivie de repousses plus ou moins importantes. L'importance du bourgeonnement varie avec les espèces parasites et la période de la coupe. Dans tous les cas, le bourgeonnement se fait à partir du moignon de tige épargné. Il va s'en suivre que l'importance quantitative des rejets sera fonction du diamètre de la base de la tige détruite. La morphologie et le fonctionnement du point de fixation de T. globiferus et T. ophiodes sur l'hôte favorisent un important bourgeonnement (Fig. 2). Au site d'implantation, l'hôte et le parasite semblent avoir une croissance stimulée et synchronisée. Tout se passe comme si l'hôte croissait pour "engloutir" l'intrus qui, lui, élargit sa base pour ne pas se faire détruire. Il en résulte une hypertrophie des tissus en forme de coupe constituée de deux hémisphères. La partie supérieure de cette coupe est constituée entièrement des tissus du parasite d'où partent les nombreuses tiges de la touffe (Fig. 2).

Chez A. dodoneifolius, la morphologie de l'haustorium est telle que le nombre de repousses après la coupe est généralement faible. La partie extérieure de l'hypertrophie provoquée par l'implantation est constituée de tissus de l'hôte. La tige, généralement unique du parasite, s'y insère par une base relativement peu élargie par rapport à celle de T. globiferus, d'où la faible repousse après la coupe.

L'hypertrophie du point de fixation d'A. dodoneifolius sur l'hôte est constituée par un enchevêtrement de tissus des 2 plantes si bien qu'aucun drageonnement n'y a encore été observé, contrairement à ce qui est décrit chez T. globiferus (Boussim 2002). 

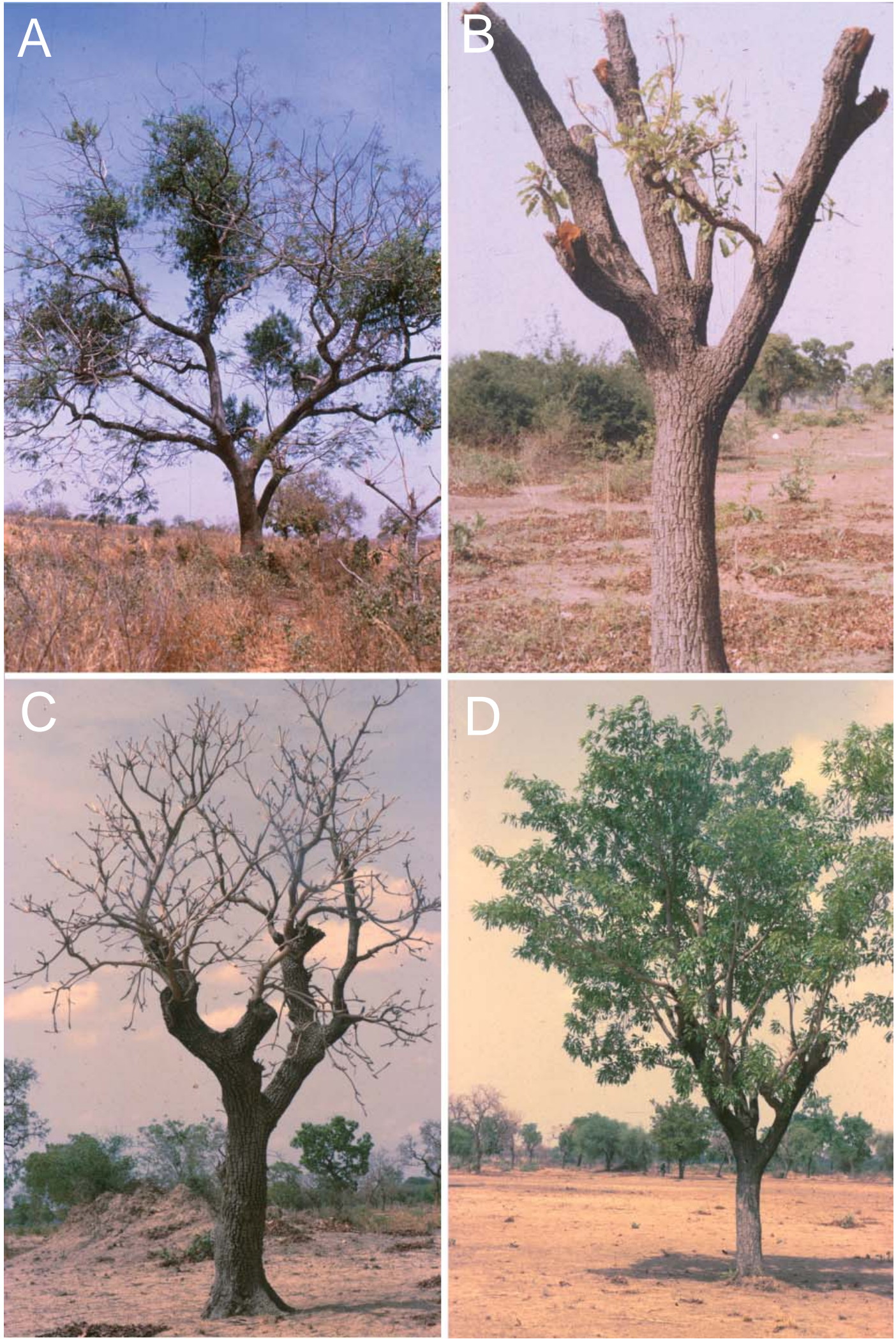

Fig. 1 : Résultat de la coupe à l'échelle de l'arbre.

A : arbre fortement parasité par Agelanthus dodoneifolius.

B : branches parasitées coupées pour éliminer le parasite

$\mathrm{C}:$ trois ans après la coupe

$\mathrm{D}$ : cinq ans après la coupe l'arbre rajeuni a retrouvé un aspect normal.

Fig. 1: Effect of the removal of infected branches

A: a tree heavily parasited by Agelanthus dodoneifolius

$B$ : infected branches cut

C: three years later

D: five years later the tree has a normal appearence. 

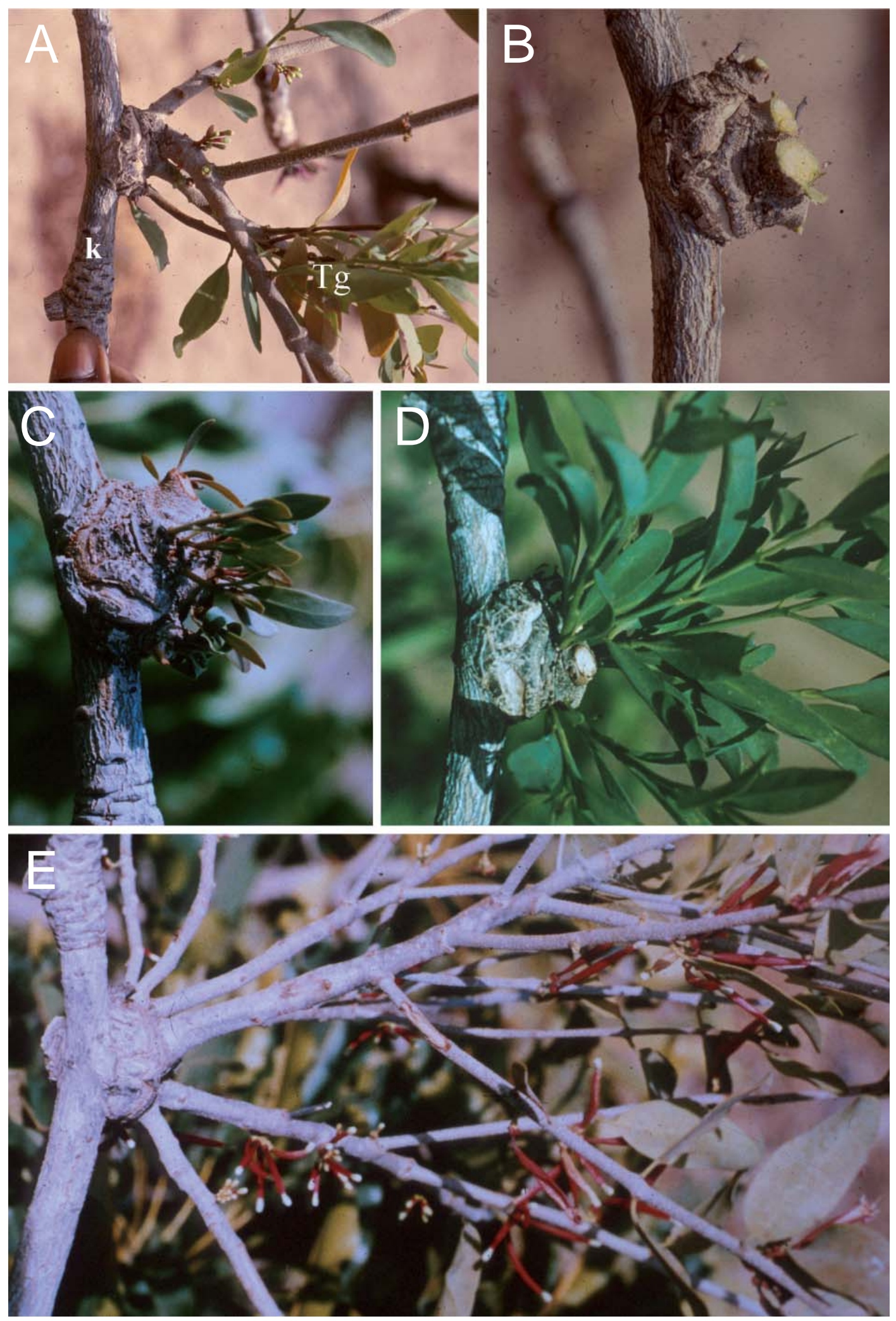

Fig. 2 : Coupe des tiges du parasite

A : touffe de Tapinanthus globiferus comportant quatre tiges.

$\mathrm{B}$ : les 4 tiges de la touffe sont coupées à ras.

C : 1 mois après la repousse est importante.

$\mathrm{E}: 2$ mois après.

F : 6 mois après. Le nombre de tiges a augmenté et le parasite a repris sa production de graines. $\mathrm{k}:$ karité; $\mathrm{Tg}: \mathrm{T}$. globiferus

Fig. 2: Cutting the twigs of the parasite

A: Tapinanthus globiferus plant with four twigs

B: the four twigs have been cut

C: one month later a remarkable resprouting can be observed

D: two months later

E: six months later the number of twigs has increased and the parasite produces seeds 
Tableau 1: Repousses de Loranthaceae après coupe le leurs tiges | Table 1: Resprouting of the Loranthaceae after cutting of their twigs

\begin{tabular}{|l|l|c|c|c|c|c|c|c|}
\hline $\begin{array}{l}\text { Date de } \\
\text { Coupe }\end{array}$ & Espèces Parasites & $\begin{array}{l}\text { Nbre tiges } \\
\text { coupée }\end{array}$ & $\begin{array}{l}\text { Rep. 3e } \\
\text { semaine }\end{array}$ & $\begin{array}{l}\text { Rep. 6e } \\
\text { semaine }\end{array}$ & $\begin{array}{l}\text { Rep. 9e } \\
\text { semaine }\end{array}$ & $\begin{array}{l}\text { Rep.12e } \\
\text { semaine }\end{array}$ & $\begin{array}{l}\text { Rep.15e } \\
\text { semaine }\end{array}$ & $\begin{array}{l}\text { Rep.18e } \\
\text { semaine }\end{array}$ \\
\hline $24-04-99$ & A. dodoneifolius & 4 & 9 & 9 & 9 & 6 & 6 & 6 \\
\hline $24-04-99$ & T. globiferus & 6 & 16 & 23 & 23 & 23 & 21 & 21 \\
\hline $24-04-99$ & T. ophiodes & 5 & 10 & 12 & 14 & 11 & 11 & 11 \\
\hline $15-06-99$ & A. dodoneifolius & 4 & 11 & 7 & 5 & 6 & 6 & 6 \\
\hline $15-06-99$ & T. globiferus & 9 & 13 & 23 & 24 & 24 & 25 & 23 \\
\hline $15-06-99$ & T. ophiodes & 4 & 6 & 11 & 11 & 12 & 12 & 12 \\
\hline $13-11-99$ & A. dodoneifolius & 4 & 6 & 1 & 0 & 0 & 0 & 0 \\
\hline $13-11-99$ & T. globiferus & 5 & 5 & 6 & 4 & 3 & 4 & 4 \\
\hline $13-11-99$ & T. ophiodes & 3 & 2 & 2 & 4 & 3 & 3 & 3 \\
\hline
\end{tabular}

Nbre= nombre; $\mathbf{R e p}=$ nombre de repousse; $\mathbf{A}=$ Agelanthus; $\mathbf{T}=$ Tapinanthus

Tableau 2: Résultats de l'application des herbicides | Table 2: Effects of the application of herbicides

\begin{tabular}{|l|c|c|c|c|c|}
\hline Herbicides & Dose & Nombre touffes & Touffes détruites & Regénération & \% destruction \\
\hline Témoin & T0 & 9 & 0 & 0 & 0 \\
\hline Glyphosate & T1 & 17 & 12 & 0 & 70.5 \\
\hline Glyphosate & T2 & 20 & 20 & 0 & 100 \\
\hline Glyphosate & T3 & 13 & 8 & 0 & 61.5 \\
\hline Glyphosate & T4 & 6 & 5 & 0 & 83.3 \\
\hline Glyphosate & T5 & 33 & 33 & 3 & 90.9 \\
\hline 2,4-D & T1 & 18 & 18 & 2 & 88.8 \\
\hline 2,4-D & T2 & 29 & 29 & 0 & 100 \\
\hline 2,4-D & T3 & 29 & 28 & 0 & 96.5 \\
\hline 2,4-D & T4 & 36 & 36 & 0 & 100 \\
\hline 2,4-D & T5 & 11 & 11 & 1 & 90.9 \\
\hline
\end{tabular}

La vitesse de régénération et du développement des repousses est fonction de la période de l'année. La période allant de novembre à la mi-mars correspond à une phase de ralentissement de l'activité végétative de la plupart des plantes au Sahel, dont les Loranthaceae et leur principal hôte, le karité. Durant cette période, souffle dans ladite zone l'Harmattan, un vent sec et asséchant et l'humidité atmosphérique est à son plus bas niveau. La plupart des plantes perdent leurs feuilles et ralentissent leur développement aérien. La vitesse de bourgeonnement des touffes de Loranthaceae recépées durant cette période est faible à nulle. C'est parmi les essais de coupe mis en place en novembre que nous avons enregistré l'élimination définitive du parasite (Tab.1).

A l'opposé, les coupes effectuées en avril ont donné de nombreux rejets qui sont florifères en octobre de la même année (Fig. 2).

La destruction du parasite par la coupe du rameau hôte en amont du point de fixation du parasite est définitive. Aucune repousse de la plante parasite n'est possible après l'élimination de l'appareil aérien et du système endophytique.

\subsection{Lutte chimique}

La réaction des parasites aux traitements a été rapide. Les premiers symptômes sont apparus avant même le premier relevé. Au fil des différents relevés, les effets suivants ont été observés :

- noircissement des feuilles,

- brûlure des feuilles,

- chute des feuilles et des fleurs,

- dessèchement progressif des rameaux et des fruits.

Le Tableau 2 traduit une forte réaction des parasites aux traitements. Les pourcentages de destruction des touffes de parasite varient entre 60 et $100 \%$. La destruction des touffes tend vers $100 \%$ avec le 2,4-D. Quant au glyphosate, les dégâts sont compris entre $60 \%$ et $100 \%$ selon les doses utilisées.

Le Tableau 3 montre également que le nombre de pulvérisation a une influence sur la réaction au traitement. Cela se traduit par l'accroissement du taux de destruction de T1 à T5 pour l'ensemble des herbicides.

En ce qui concerne l'action spécifique des produits utilisés, le glyphosate semble présenter une lenteur de réaction par rapport au 2,4-D. En effet, un mois après le traitement, on n'a pas constaté une perte importante, sauf chez A. dodoneifolius qui semble montrer une forte sensibilité aux produits. 
Tableau 3: Effet mode de traitement | Table 3: Effect of the way of treatment

\begin{tabular}{|c|c|c|c|c|c|}
\hline Herbicides & Doses & Mods & Nbre touffes & $\begin{array}{l}\text { Nbre touffes dé- } \\
\text { truites }\end{array}$ & $\%$ destruction \\
\hline Témoin & T0 & 0 & 9 & 0 & 0 \\
\hline Glyphosate & $\mathrm{T} 1$ & 1 & 7 & 6 & 85.7 \\
\hline Glyphosate & $\mathrm{T} 1$ & 2 & 10 & 8 & 80 \\
\hline Glyphosate & $\mathrm{T} 2$ & 1 & 10 & 8 & 80 \\
\hline Glyphosate & $\mathrm{T} 2$ & 2 & 10 & 7 & 70 \\
\hline Glyphosate & $\mathrm{T} 3$ & 1 & 6 & 6 & 100 \\
\hline Glyphosate & T3 & 2 & 7 & 5 & 71.4 \\
\hline Glyphosate & $\mathrm{T} 4$ & 1 & 3 & 3 & 100 \\
\hline Glyphosate & $\mathrm{T} 4$ & 2 & 3 & 3 & 100 \\
\hline Glyphosate & T5 & 1 & 18 & 9 & 50 \\
\hline Glyphosate & $\mathrm{T} 5$ & 2 & 15 & 6 & 39.9 \\
\hline $2,4-\mathrm{D}$ & $\mathrm{T} 1$ & 1 & 9 & 9 & 100 \\
\hline $2,4-\mathrm{D}$ & $\mathrm{T} 1$ & 2 & 9 & 9 & 100 \\
\hline $2,4-\mathrm{D}$ & $\mathrm{T} 2$ & 1 & 13 & 11 & 84.6 \\
\hline $2,4-\mathrm{D}$ & $\mathrm{T} 2$ & 2 & 16 & 14 & 87.5 \\
\hline $2,4-\mathrm{D}$ & $\mathrm{T} 3$ & 1 & 16 & 14 & 87.5 \\
\hline $2,4-\mathrm{D}$ & $\mathrm{T} 3$ & 2 & 13 & 13 & 100 \\
\hline $2,4-\mathrm{D}$ & $\mathrm{T} 4$ & 1 & 17 & 17 & 100 \\
\hline $2,4-\mathrm{D}$ & $\mathrm{T} 4$ & 2 & 18 & 17 & 94.4 \\
\hline $2,4-\mathrm{D}$ & T5 & 1 & 5 & 5 & 100 \\
\hline $2,4-\mathrm{D}$ & T5 & 2 & 6 & 6 & 100 \\
\hline
\end{tabular}

Un dessèchement localisé des feuilles du karité atteintes par le produit a été observé. Cet effet des herbicides sur la plante hôte a été minimisé par le fait qu'au moment de l'application des produits, le karité était en défeuillaison. A la reprise de la végétation, les branches de karité portant les touffes traitées ont donné des feuilles et fleurissent normalement comme celles non traitées.

\subsection{Recherche de variétés résistantes}

Un seul arbre (Fig. 3) des cinq dont les branches ont été artificiellement infestées par les baies des parasites ne porte aucune plantule parasite et semble présenter par conséquent une sensibilité nulle. La germination des Loranthaceae étant indépendante de la nature de l'hôte, les graines viables utilisées ont germé. Des pénétrations et apparitions des premières feuilles ont été parfois observées, ce qui n'est pas extraordinaire chez les Loranthaceae qui pénètrent leurs propres tissus. Mais aucun développement n'a dépassé le stage de 2 feuilles chez le pied en question, confirmant ainsi l'existence d'une barrière empêchant le développement du parasite. La même observation a été faite sur le Striga par KUIPER et al. (1998). La résistance au parasite ne s'exprime pas au niveau de la germination ni de l'attachement. C'est le développement subséquent des plantules qui est entravé.

Ce résultat et le cas de certains cultivars de peupliers avec le gui européen (HARIRI et al. 1990) nous confortent dans notre projet de privilégier la voie génétique dans nos prochains travaux, sans occulter les autres voies, dans la lutte contre les Loranthaceae.

\subsection{Ennemis naturels}

Quatre types d'intervention des ennemis naturels des Loranthaceae ont été identifiés (Fig. 3) :

- Destruction des graines par des oiseaux. Les embryons des graines collées par le petit barbu à front jaune (Pogoniulus chrysonocus) sont extraits et mangés par d'autres oiseaux, probablement, granivores. Les restes de ces graines (albumen et téguments) jonchent les branches environnant la touffe fructifère. Des observations similaires ont été rapportées par Sallé et al. (1993) sur Viscum album.

- Destruction du système haustorial par une larve d'un papillon. Sur le terrain, nous avons observé des touffes de $T$. ophiodes en train de dépérir. En recherchant la raison du flétrissement, nous avons noté la présence à la base de la tige, d'une grosse larve blanche, identifiée comme étant celle d'un papillon. Cette larve creuse une large galerie dans le système endophytique du parasite, ce qui affecte son fonctionnement normal et entraîne le dessèchement et la chute du parasite.

- Destruction foliaire par des insectes. A Saria, nous avons observé un peuplement important de T. ophiodes parasitant Acacia olosericea, et à Pô des touffes de T. globiferus sur karité, dont le feuillage et les bourgeons terminaux sont systématiquement détruits par un petit insecte noir 

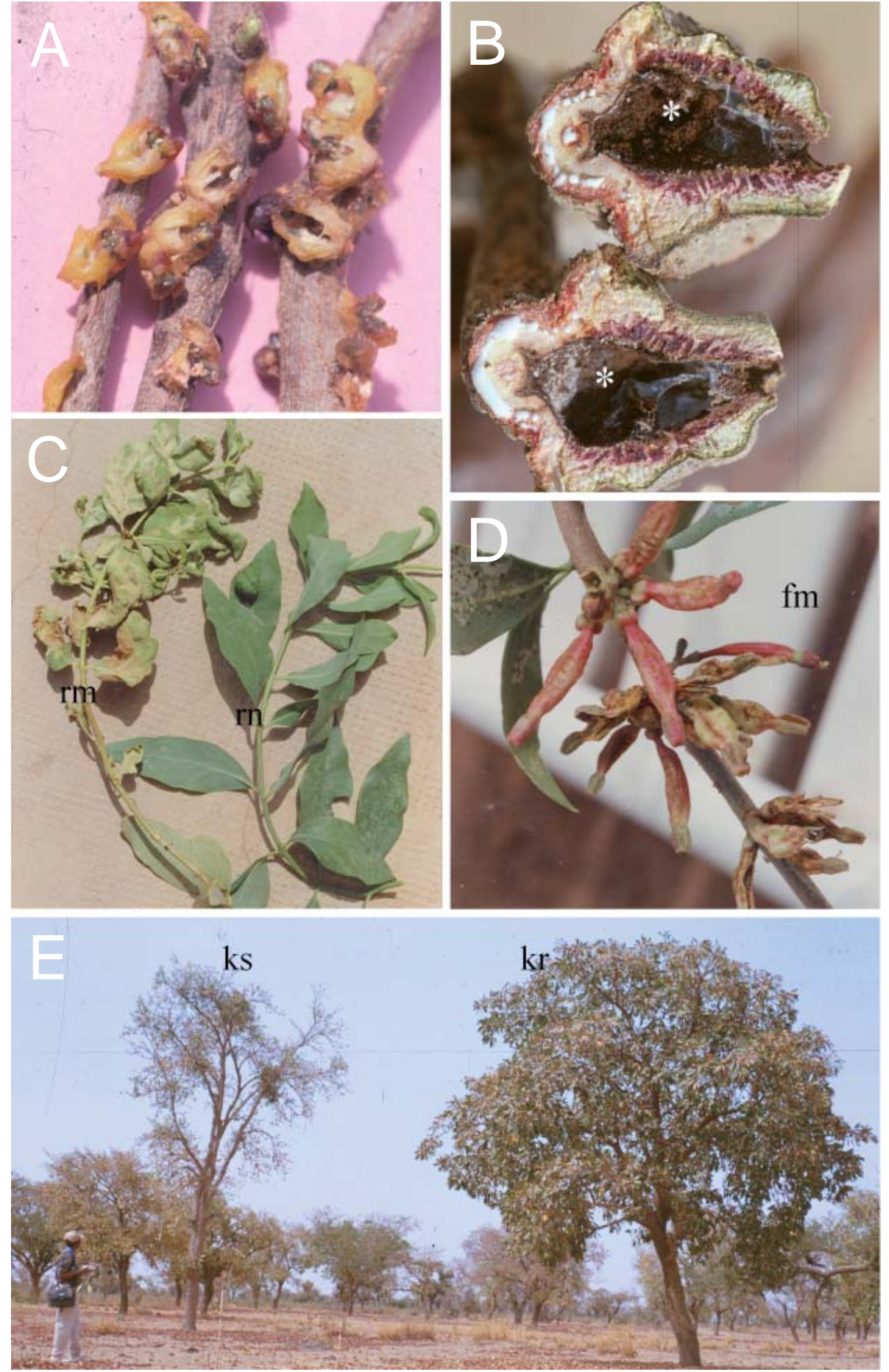

Fig. 3 : Perspective de lutte biologique : ennemis naturels et résistance variétale

A : restes d'albumen et des téguments de la graine sans embryon.

B : large galerie creusée dans l'haustorium de Tapinanthus ophiodes par la larve d'un papillon.

B : destruction des jeunes feuille du parasite par des insectes qui y pondent

D : destruction des boutond floraux de Tapinanthus globiferus par des insectes qui y deposent leurs œufs.

E : Karité résistant $(\mathrm{kr})$ cohabitant (distance de séparation $=22 \mathrm{~m}$ ) avec un karité sensible $(\mathrm{ks})$ fortement attaqué par Agelanthus dodoneifolius et Tapinanthus globiferus (flèches) $\mathrm{h}$ : hôte ; $\mathrm{p}$ : parasite ; fm : fleurs malades ; $\mathrm{kr}$ : karité résistan

ks karité sensible; rm : rameau malade ; rn : rameau sain ;

Fig. 3 : Perspectives of biological pest control : natural enemies and resistant varieties

A : Fruits destroed by birds.

B : haustorium of Tapinanthus ophiodes infected by a larve of a butterfly.

$\mathrm{B}$ : destruction of leaves by insects

D : destruction of the flowers of Tapinanthus globiferus by insectes.

$\mathrm{E}$ : resistent Karité (kr) neighboring (distance $22 \mathrm{~m}$ ) a sensible karité (ks) highly infected by Agelanthus dodoneifolius and Tapinanthus globiferus. 
volant. D'importantes colonies d'insectes déposent leur ponte dans les feuilles et les bourgeons.

- Attaque florale. Dans certaines zones du Burkina Faso, nous avons observé de nombreuses touffes de T. globiferus sur karité dont les fleurs portent des malformations les rendant incapables d'évoluer en fruits. L'ouverture forcée (elles ne s'ouvrent pas naturellement) des fleurs révèle la présence de petits insectes ailés et de leurs œufs. Ainsi, les organes reproducteurs de la fleur sont détruits par les insectes.

\section{Conclusion}

Les différents essais de contrôle des Loranthaceae sur le karité effectués lors du présent travail ont fourni beaucoup d'informations et permis de tirer des enseignements.

La lutte mécanique est la plus simple et la plus à la portée de la population rurale, même si elle comporte un certain nombre d'ennuis (l'accessibilité du parasite, l'étendue de l'infestation). Sa réussite dépend cependant du niveau et de la période de la coupe. Une coupe de la branche parasitée qui inclurait le système endophytique du parasite, élimine définitivement celui-ci, quelque soit le période. Si la coupe ne devait pas concerner l'endophyte, la période de repos végétatif de l'hôte est conseillée pour son application.

La méthode de lutte chimique, déjà courante chez les parasites de racines, a donné des résultats positifs. Mais les ennuis qu'elle comporte (coût des produits chimiques et du matériel, technique d'application, étendue de l'infestation, etc.) sont tels qu'il nous paraît raisonnable d'entreprendre des études complémentaires avant de l'envisager comme voie de contrôle des Loranthaceae sur le karité.

Bien que la recherche de variétés résistantes ne soit pas encore étendue sur tout le pays, les premiers résultats sont encourageants. Avec la maîtrise de la technique de multiplication végétative et la domestication du karité en cours, la sélection de variétés résistantes de l'espèce aux Loranthaceae nous semble être une voie de lutte à prospecter avec beaucoup d'espoir, même si son effet ne peut être perçu qu'à long terme.

Les résultats de nos investigations montrent que, comme le Striga (Williams \& Caswell 1959, Greathead 1984, Greathead \& Milner 1971, Bashir 1987, Bashir \& Musselman 1984, Markham 1985, Traoré et al. 1991) et la Cuscuta (AgRAWAL 1983), les Loranthaceae sur le karité ont des prédateurs naturels qui sont principalement d'espèces d'oiseaux et d'insectes et qu'il y a donc une possibilité de lutte biologique. Mais là aussi, une éventuelle utilisation de ces animaux comme moyen de contrôle du parasite, ce qui aurait pour mérite d'éviter le problème de toxicité des produits chimiques, nécessite au préalable une étude de base de la biologie et d'écologie de ces espèces.

\section{REMERCIEMENTS}

Les auteurs remercient le projet ENRECA-Botanique/DANIDA et l'INERA/CNRST à travers le CRREA Centre-Ouest Saria pour leur appui financier.

\section{BIBLIOGRAPHIE}

AgrawAL BD (1983): Life history of Cuscuta gall weevil Smicronyx roridus Mashl. (Coleoptera : Curculionidae). Cecidol. Int. 5: 41-46.

Baillon F, Chamel A, Fer A \& Frochot H (1988): Lutte chimique contre le gui (Viscum album L.). Pénétration, transport, efficacité de deux herbicides phloème-mobiles (2,4-DB et glyphosate). - Ann. Sci. 45(1): 1-16.

BASHIR OM (1987): The potential for biological control of witchweeds. - In Musselman, LJ (ed.): Parasitic weeds in agriculture. Volume 1: Striga. Boca Raton, Florida. CRC Press, 183-206.

BAshir OM \& Musselman LJ (1984): Some natural enemies of Striga hermonthica in the Sudan. - Trop. Pest Manag. 30: 211-212.

Boussim IJ (2002): Les phanérogames parasites du Burkina Faso: Inventaire, taxonomie, écologie et quelques aspects de leur biologie. Cas particulier des Loranthaceae parasites du karité. - Thèse de Doctorat d'Etat es Sci. Naturelles. Université de Ouagadougou, Ouagadougou, 306 p.

Boussim IJ (1991): Contribution à l'étude des Tapinanthus parasites du karité au Burkina Faso. - Thèse de doctorat de 3e cycle, Université de Ouagadougou, Ouagadougou 152 p.

Boussim IJ, SAllÉ G \& GuINKo S (1993a): Tapinanthus parasite du karité au Burkina Faso. 1e partie: Identification et distribution. - Bois Forêts Tropiques 238: 53-58.

Boussim I J, SAllé G \& GuINKo S (1993b): Tapinanthus parasite du karité au Burkina Faso. 2e partie: Phénologie, biologie et dégâts. - Bois Forêts Tropiques 238: 59-65.

CleRK GC (1978): Tapinanthus bangwensis in Cola plantation in Ghana. - PANS 24(1): 57-62.

COMPAORÉ E (1986): Etude de la résistance au Striga gesnerioides chez quelques cultivars de niébé (Vigna unguiculata (L.) Walp.). - Mém. d'Ing. de Dével. Rural. Inst. Sup. Polytech. Université de Ouagadougou, Ouagadougou, 69 p.

DJIGUEMdé FA (1985): Contribution à la lutte contre Striga hermonthica (Del.) Benth. sur le sorgho. Mém. d'Ingénieur du Développement Rural. Inst. Sup. Polytech. Université de Ouagadougou, Ouagadougou, 74 p.

Doggett H (1952): Botanist's Ann. Rep., 1950 Season, Tanganyka. - Dep. Agric. Section III: 222-244.

DogGetT H (1965): Striga hermonthica on Sorghum in East Africa. - J. Agri. Sci. 65: 183-194.

Frochot H \& Delabraze P (1979): Efficacité d'herbicides du groupe des aryloxyacides sur le gui du sapin. - 10e conférence COLUMA, Versailles 1, 157-165.

Frochot H, Pitsch M, Wehrlen L (1983): Efficacité d'herbicides sur le gui des feuillus (Viscum album Mali) installé sur peuplier, Maison de l'UNESCO, Paris, 165 p.

Greathead DJ (1984): The natural enemies of Striga spp. and the prospection for their utilization as biological control agents. - Workshop Biol. Control Striga: 133-160.

GreatheAd DJ \& Milner JFD (1971): A survey of Striga spp. and their insect natural enemies in East Africa with a discussion on the possibilities of biological control. - Trop. Agric. 48: 111-124.

Guyot J \& NTAWANGa OMAnda E (1998): Note sur la sensibilité de six clones d'hévéa à Phragmenthera capitata. Plantations, Recherche, Développement. 5(5). 
HARIRI EB (1989): Polyphenols et résistance au gui (Viscum album L.) étude histocytochimique et analytique. - Doctorat de l'Université Pierre et Marie Curie (Paris VI), Paris, 179 p.

Hariri E B, SAllé G \& Andary C (1990): Mécanisme de résistance de 4 cultivars de peuplier en réponse à l'attaque du gui (Viscum album L.). - C.R. Acad. Sci. Paris 311(3): 439-444.

Kuiper E, Groot A, Noordover CME, Pieterse AH \& VERKLEIJ ACJ (1998): Tropical grasses vary in their resistance to Striga aspera, S. hermonthica, and their hybrids. - Can. J. Bot. 76: 2131-2144.

MAÏGA AY (1988): Action thématique sur la mortalité du karité (Butyrospermum paradoxum) (Gaertn. f.) Hepper dans la région de Ségou. - Rap 2e mission CCE.

MAÏGA AY (1989): Actions thématiques sur la mortalité du karité Butyrospermum paradoxum (Gaertn f.) Hepper dans la région de Ségou. Phase de prolongation. - Rapport de la 3e mission CCE. Répartition géographique des Loranthaceae parasites du karité, $36 \mathrm{p}$.

MARKHAM RH (1985): Possibilities of biological control of Striga species in Sahel. Permanent interstate Committee for Drought Control in the Sahel. - Mission rapport. CILSS, Ouagadougou, $20 \mathrm{p}$.

MinINGOU A (1985): Criblage de variétés de sorgho pour la résistance au Striga hermonthica (Del.) Benth. - Mém. d'Ing. Dével. Rural. Inst. Sup. Polytech. Université de Ouagadougou, $78 \mathrm{p}$.

OLIVIER A (1996): La relation entre Striga hermonthica et ses hôtes: une synthèse. - an. J. Bot. 74: 119-1137.

OuÉDraogo O (1995): Contribution à l'étude de quelques Phanérogames parasites des cultures au Burkina Faso. Incidence, biologie et méthodes de lutte. - Doctorat de l’Université Pierre et Marie Curie, Paris 6, 95 p.

Room P M (1971): The mistletoe Tapinanthus bangwensis in Ghana. - Proceedings 3rd Internatinal Cocoa Research Conference, Accra, 1969: 522-527.

RuYsSEn, B (1957): Le karité au Soudan. Agronomie Tropicale 12(2): 144-172, 12(3): 279306 et 12(4): 415-438.

Sallé G, Boussim IJ, Raynal-Roques A \& Brunck F (1990): Le karité : état de nos connaissances et perspectives de recherche. Séminaire sur la physiologie des arbres et arbustes en zones arides. Paris-Nancy, 20 mars au 6 avril 1990, 17 p.

Sallé G, Frochot H \& Andary C (1993): Le gui. - Recherche 24: 1334-1342.
TRAORÉ D (1994): Lutte contre les plantes vasculaires parasites de la Côte d'Ivoire. Cas des Départements de Korhogo, Boundiali, Ferkessédougou et Tengréla. - Rapport annuel de PEP A.I.S.A.-CI, 71 p.

TrAoré D \& DA K (1995): Lutte contre les plantes vasculaires parasites du karité et du néré dans le Nord de la Côte d'Ivoire. Cas des Départements de Korhogo, Boundiali, Ferkessédougou et Tengréla. - Rapport annuel de PEP A.I.S.A.CI, $34 \mathrm{p}$.

TRAORÉ D \& DA K (1996): Lutte contre les plantes vasculaires parasites du karité et du néré dans le Nord de la Côte d'Ivoire. Cas des Départements de Korhogo, Boundiali, Ferkessédougou et Tengréla. - Rapport annuel de PEP A.I.S.A.CI, $97 \mathrm{p}$.

Traoré D \& DA K (1997): Lutte contre les plantes vasculaires parasites du karité et du néré dans le Nord de la Côte d'Ivoire. Cas des Départements de Korhogo, Boundiali, Ferkessédougou et Tengrela. - Rapport annuel de PEP A.I.S.A.CI, $16 \mathrm{p}$.

TRAORÉ D (1999): Lutte biologique intégrée contre Striga hermonhica par la manipulation de Smicronyx spp. et Fusarium oxysporium. - Mém. Ing. Dévelop. Rural, Univ. Polytech. Bobo Dioulasso, $53 \mathrm{p}$.

Traoré D, Vincent C \& SÉRÉ A (1991): Prospects for biological control of Striga (witchweed) in Burkina Faso. In RANSOM JK, Musselman LJ, Worsham, AD \& PARKER $\mathrm{C}$ (eds.): Proc. 5th International Symposium on parasitic weeds. CIMMYT, Nairobi, Kenya, 328 p.

Williams CN \& CASWell G H (1959): An insect attacking Striga. - Nature 184, 1668 p.

YONLI D (1999): Lutte biologique contre Striga hermonthica (Del.) Benth. à base de Fusarium oxysporum Schel. Emend. Snyder et Hans. - Mémoire de D.E.A. Université de Ouagadougou, Ouagadougou. 71 p.

\section{AdResse DES AUTEURS}

\section{Joseph I. Boussim}

\section{Nayéré Médah}

Laboratoire de Biologie et Ecologie Végétales, UFR Sciences de la Vie et de la Terre, Université de Ouagadougou,

03 BP 7021 Ouagadougou 03, Burkina Faso. 